\title{
Centers of structures in electromagnetism--a critical analysis
}

\section{Appel-Hansen, Jørgen}

\section{Published in:}

I E E E Transactions on Antennas and Propagation

Link to article, DOI:

10.1109/TAP.1982.1142861

Publication date:

1982

Document Version

Publisher's PDF, also known as Version of record

Link back to DTU Orbit

Citation (APA):

Appel-Hansen, J. (1982). Centers of structures in electromagnetism--a critical analysis. I E E E Transactions on Antennas and Propagation, 30(4), 606-610. https://doi.org/10.1109/TAP.1982.1142861

\section{General rights}

Copyright and moral rights for the publications made accessible in the public portal are retained by the authors and/or other copyright owners and it is a condition of accessing publications that users recognise and abide by the legal requirements associated with these rights.

- Users may download and print one copy of any publication from the public portal for the purpose of private study or research.

- You may not further distribute the material or use it for any profit-making activity or commercial gain

- You may freely distribute the URL identifying the publication in the public portal

If you believe that this document breaches copyright please contact us providing details, and we will remove access to the work immediately and investigate your claim. 


\title{
Centers of Structures in Electromagnetism-A Critical Analysis
}

\author{
JØRGEN APPEL-HANSEN
}

\begin{abstract}
Some principles for finding reference points or centers of structures in electromagnetism are outlined. It is pointed out that the centers which are found depend on arbitrary choices. Since some of the principles are based on Friis's transmission formula and the radar equation, these are given in forms suitable for finding centers. The use of correction factors and near-field parameters are outlined. In particular, proper use of different near-field radar cross sections for plates and spheres are outlined.
\end{abstract}

\section{INTRODUCTION}

W HEN COMMUNICATION from one structure to another structure takes place, all parts of the two structures contribute more or less actively. Based on the solution of Maxwell's equations and by defining several magnitudes, communication relations can be derived. The most often used relations in practice are Friis's transmission formula for oneway communication and the radar equation for two-way communication. When using these relations, it is necessary to insert a distance between the structures involved. This creates an ambiguity problem because the structures are not point objects. For distances between the structures that are sufficiently large relative to the actual dimensions of the structures and the wavelength, the choice of reference points is not critical. At shorter distances, e.g., on test ranges such as anechoic chambers, the results obtained by using the communication relations may depend significantly on the chosen reference points.

The present work was initiated from the observation that during calibration of a radar cross section measurement set up on a $10-\mathrm{m}$ range using a $20-\mathrm{cm}$ diameter sphere, the results undergo a change of about $0.2 \mathrm{~dB}$ with a change in distance of $10 \mathrm{~cm}$, e.g., if the crown point on the front surface of the sphere is used as a reference point instead of its geometric center [1].

The purpose of the present paper is to outline several principles which may be used to find proper reference points for structures in electromagnetism. Since it is often required that some electromagnetic properties are described in a simple manner by using the reference points, they are called centers of the structures. The fact that the final results depend on arbitrary choices is not unusual in electromagnetism; common examples are the impedance of a waveguide and the $Q$ of a resonator.

As it will be apparent from the following sections, nearfield parameters are parameters which use concepts and symbols analogous to the corresponding true far-field parameters. True far-field parameters are parameters found in the limit as the distance tends to infinity, e.g., gain, receiving cross section, and radar cross section. Correction factors are factors to true far-field parameters taking into account the finite distance in a communication problem. The product

Manuscript received January 23, 1981; revised May 13, 1981.

The author is with the Electromagnetics Institute, Technical University of Denmark, DK-2800 Lyngby, Denmark. of a correction factor and the corresponding true far-field parameter may be defined as a near-field parameter. In measurements, systematic errors will be present in case correction factors are not used [4].

In Section II some examples of centers used in electromagnestism are given. The arbitrary choices adopted in the principles used to find the centers are pointed out. In order to make a more detailed discussion of some of the principles, a general communication relation is established in Section III. In Section IV this relation is put in a form analogous to Friis's transmission formula and near-field correction factors are discussed. In Section V-A it is demonstrated that two-way transmission may be considered as a special case of one-way transmission. Furthermore, a two-way transmission formula is cast in a form analogous to the radar equation. In Sections $\mathrm{V}-\mathrm{B}$ and $\mathrm{V}-\mathrm{C}$ different expressions for near-field radar cross sections of large plates and spheres are discussed. For further details the reader is referred to [3].

\section{PRINCIPLES FOR FINDING CENTERS}

The field around a radiating structure is usually described in phase and amplitude in a smaller or larger region of space. The region may be specified by making use of a reference point which may be called a center of radiation. In the case of an infinitesimal radiator such as a Hertzian dipole, it is natural (but not required) to suggest the position of the dipole as the center of radiation from which information is transformed to other points in space. If the radiator has some extent, it is not straightforward to indicate a center of radiation. To do this a principle to be used has to be suggested.

From a mathematical point of view it is natural to apply a principle which may lead to some computational convenience. Let us consider series expansion of fields. It is wellknown that the number of significant terms depends on the size of the radiating structure and the position of the origin of the applied coordinate system relative to the radiating structure. Therefore, the center of radiation may be defined as the origin of the coordinate system which gives the minimum number of significant terms in the expansion series. Let us refer to this principle of finding the center of radiation as the minimum-number principle. In order to apply the principle an arbitrary choice of criterion has to be made in the assessment of when a significant number of terms is reached.

Let us illustrate the minimum-number principle by a consideration of spherical wave expansions. In this case the number of significant terms depends on the maximum value of a modal index $n$ which is required in order to have a satisfactory (specified arbitrarily) representation of the radiated field. It is found that the expansion can often be truncated at $n=k a$, where $a$ is the radius of the smallest sphere enclosing the antenna and $k$ is the wavenumber [5], [7]. This fundamental observation leads to the suggestion that the first choice of the position of the origin of the coordinate system should be such that a sphere having its center at the 
origin and enclosing the radiating structure has a minimum radius. According to the truncation criterion $n=k a$, such a choice is likely to give a small number of significant terms in the field expansion. From the physical geometry of the radiator, it is usually a simple matter to make the first choice. Then an optimization process may follow in which the origin may be varied about the first choice in order to minimize the number of significant terms in accordance with the minimumnumber principle.

It should be mentioned that in the first choice of the position of the origin, the current distribution on the radiating structure may be taken into account. For example, if this has a well-defined aperture, the first choice of the position of the origin could be made so that the sphere having its center at the chosen origin and enclosing the aperture has a minimum radius. In fact in the case of horn antennas, it has been demonstrated that when the origin is chosen in the middle of the aperture plane, fewer spherical waves are required than when the origin is located at the phase center of the horn (defined such that the phase pattern is flat over the main beam) [15].

It is interesting to note that in near-field scanning techniques, it is often said that the center of rotation should be chosen conveniently in or close to the structure. From the above it is seen that in case the center of rotation is chosen as the center of radiation found by applying the minimumnumber principle, computational convenience can be expected. In practice, mechanical stability and alignment procedures may also influence the choice [4].

As another example of finding the center of radiation let us consider the principle used in determinig the phase center of a radiating structure. The principle adopted is to find a point so that the phase of the far-field is relatively constant over some spherical segment [8]. The point which is found is referred to as the phase center and is considered as the center from which radiation emanates. It is understood that if the phase center is determined experimentally using conventional far-field techniques, it depends on the test distance. Furthermore, it must depend on the size of the spheriical sector considered. In addition to this, since measurements cannot be expected to give a perfect constant phase, the obtained phase center will also depend on the principle used to choose the final phase variation over the spherical sector considered. Only for linear antennas and arrays that have special symmetrical properties in their current distribution, phase centers exist [10] in theory.

As an additional example of finding a center of radiation, let us consider a principle used to find an amplitude center. The principle adopted here is to find a point from which the field amplitude varies relatively closely to the inverse distance variation in a specified direction over some distance range. Thus the obtained amplitude center depends on the choice of several parameters used in applying the principle, e.g., in case of investigation of surface wave propagation over low density medium [2]. The choice of distance range may be made, e.g., by considering the series in powers of inverse distance in which coupling between antennas can be expressed [17].

In order to illustrate additional principles related to reflection centers, let us consider a set up for scattering measurements. Here, a radar illuminates a scattering object. As a result, a current distribution is generated on the test object and a signal is detected by the radar. It is said that the object scatters a signal back to the radar. The magnitude of this signal is calculated by making use of the scattering cross section $\sigma$ of the object. This is defined as

$$
\sigma=\lim _{R \rightarrow \infty} 4 \pi R^{2} \frac{\left|\bar{E}_{s}\right|^{2}}{\left|\bar{E}_{i}\right|^{2}}
$$

where $\bar{E}_{i}$ is the electric field intensity of an (often) plane wave illumination of the object, and $E_{s}$ is the scattered field at a point which is characterized with respect to the object by using an appropriately chosen coordinate system. The parameter $R$ is the distance between the point and the origin of the coordinate system. It is noted that $\sigma$ is found by letting $R$ tend to infinity. Thus $\sigma$ is an example of a true far-field parameter. It is understood that the value of $\sigma$ is independent of the choice of the position of the origin of the coordinate system in or at a finite distance from the object [9].

The power $P_{r}$ received by the radar is now calculated from the radar equation as

$$
P_{r}=\frac{P_{t} G}{4 \pi R^{2}} \frac{\sigma}{4 \pi R^{2}} A
$$

where $P_{t}$ is the power transmitted by the radar, $G$ is the gain of the radar antenna, $A$ is its receiving cross section, and $R$ is the distance between the radar antenna and the object. As discussed in Section $\mathrm{V}$ two major principles can be used in order to find apparent reflections centers and corresponding scattering cross sections.

For an arbitrary antenna or scatterer the chosen principle depends on several factors. One factor is the manner in which the application of the principle is in accordance with a physi$\mathrm{cal}$ interpretation of the actual phenomena. For example, the principle of geometrical optics considers both a receiving center and a reflection center as described in Section $V$. Another factor influencing the chosen principle is that it may be required that the obtained center is valuable for a specific practical purpose. For example, in the case of the sphere, a near-field cross section corresponding to the crown point may be chosen. This facilitates the experimental calibration of a scattering measurement set up because the distance to the crown point can be easily measured with, e.g., a meter stick. The description of the different principles are given below in general terms and illustrated for simple cases. Thus it is hoped that the reader may get ideas to construct centers for other cases whenever needed.

\section{COMMUNICATION RELATION}

It is not new to consider any conveyance of information from one body in space to another body in space as a communication problem. Let us consider communication between two bodies and let us adopt the terminology used in electromagnetism.

In Fig. 1 let Body 1 be a transmitting body characterized by a point $A_{t}$ and let Body 2 be a receiving body characterized by a point $O_{r}$. The points $A_{t}$ and $O_{r}$ are points chosen arbitrarily in or at a finite distance from the bodies. Let $R\left(A_{t}, O_{r}\right)$ be the distance between the points $A_{t}$ and $O_{r}$, and let $P_{t}$ and $P_{r}$ be the transmitted and received information, respectively. Furthermore, let the ability of Body 1 to transmit information to Body 2 be denoted $G\left(A_{t}, O_{r}\right)$ and let the ability of Body 2 to receive information from Body 1 be denoted $A\left(A_{t}, O_{r}\right)$. Between the parameters defined above, it is postulated that 


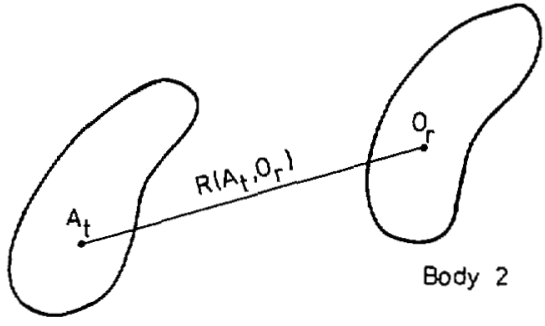

Body $i$

Fig. 1. Communication problem.

there exists a communication relation

$$
f\left\{P_{t}, P_{r}, R\left(A_{t}, O_{r}\right), G\left(A_{t}, O_{r}\right), A\left(A_{t}, O_{r}\right)\right\}=0
$$

besides a simple relation between the coordinates of $A_{t}$ and $O_{r}$ and the distance $R$ in a properly chosen coordinate system. Let us write this relation as

$$
g\left(A_{t}, O_{r}, R\right)=0 .
$$

We are now able to make the fundamental observation that we have only two equations and generally more than two unknowns. As will be apparent from the following sections, it is based on this observation that the fundamental problems in applying Friis's transmission formula and the radar equation can be discussed.

\section{ONE-WAY TRANSMISSION}

Let us consider the case of transmission between two antennas and write the communication relation in a form analogous to Friis's transmission formula (for $A_{t} \neq O_{r}$ )

$$
P_{r}=\frac{P_{t}}{4 \pi R^{2}\left(A_{t}, O_{r}\right)} G\left(A_{t}, O_{r}\right) A\left(A_{t}, O_{r}\right) .
$$

Here the points $A_{t}$ and $O_{r}$ may be referred to as the radiating center of the transmitting antenna and the receiving center of the receiving antenna, respectively. The positions of these centers may be found using the following principle. Suppose that $P_{t}$ and $P_{r}$ are determined based on a theoretical model or based on an experiment. Furthermore, suppose that we know for arbitrarily chosen directions true far-field parameters for $G$ and $A$. By using these values for $P_{t}, P_{r}, G$, and $A$ in (5), $R\left(A_{t}, O_{r}\right)$ may be calculated. Any two points $A_{t}$ and $O_{r}$ with an interspacing equal to $R$ may be chosen as representing the transmitting and receiving centers, respectively.

Apparently, several arbitrary choices have been made above. In particular, it should be noted that some choices have to be made in the construction of a theoretical model. For an example of a theoretical model for horn antennas, see [11]. Here the distance $R\left(A_{t}, O_{r}\right)$ is chosen equal to the distance between the centers of the horn apertures. This distance together with true far-field gain values are used in Friis's transmission formula and a so-called near-field gain correction factor is included in the formula to adjust for the implications of the arbitrary choices. It is not surprising that different choices result in different correction factors as it is apparent from the literature [6], [12]. The theoretical correction factors may be used to correct measured data. However corrections may also be based on measured data, e.g., in the case of the extrapolation technique [17].

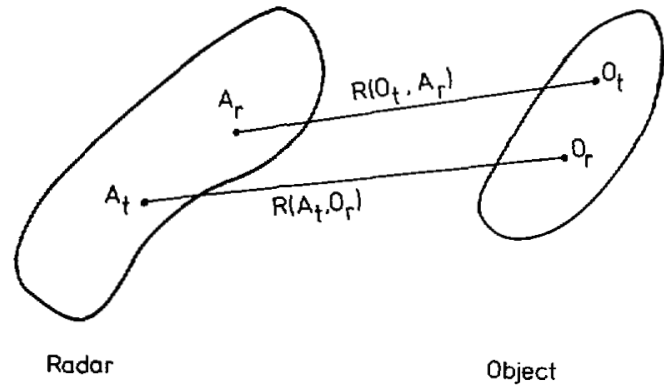

Fig. 2. Two-way transmission problem.

\section{TWO-WAY TRANSMISSION}

\section{A. General Consideration}

In case Body 1 is a radar and Body 2 is a scattering object as shown in Fig. 2, it is usually said that some of the power transmitted by the radar is reflected back to and received by the radar. Thus one may say that two-way transmission exists. However it may also be claimed that the power $P_{t}$ is transmitted from one point $A_{t}$ and this results in the power $P_{r}$ received at another point $A_{r}$. Thus in accordance with the previous sections, the communication relation is (for $A_{t} \neq A_{r}$ )

$$
P_{r}=\frac{P_{t}}{4 \pi R^{2}\left(A_{t}, A_{r}\right)} G\left(A_{t}, A_{r}\right) A\left(A_{t}, A_{r}\right) .
$$

Several problems are involved in order to introduce wellestablished quantities related to two-way transmission in this equation. In particular, the relation is meaningless in case $A_{t}$ and $A_{r}$ are chosen to be one and the same point. In fact, this is a natural choice in the case of a monostatic radar. However it should be noted that by using near-field communication relations $P_{r}$ may be found in case $A_{r}$ is chosen to be at $A_{t}$.

Let us now consider the transformation of power from $A_{t}$ and $A_{r}$ via the scattering body and let us consider the radar equation (2). Let $P_{t}$ be a quantity which is impressed under our control and let $P_{r}$ be a quantity which can be measured (or calculated). For $G, \sigma$, and $A$, we use the true far-field parameters related to arbitrarily chosen directions. Insertion of the so determined quantities into (2) may be used as a principle to find a value for $R$. Furthermore, this value of $R$ may be used to make suggestion for the center of radiation of the radar $A_{t}$, the center of reception $O_{r}$ of the scattering body, the center of reflection $O_{t}$ of the body and the center of reception $A_{r}$ of the radar, see Fig. 2. Now, let $R\left(A_{t}, O_{r}\right)$ be the distance between $A_{t}$ and $O_{r}$ and let $R\left(O_{t}, A_{r}\right)$ be the distance between $O_{t}$ and $A_{r}$. Then from a consideration of the radar equation it seems most natural to let $R\left(A_{t}, O_{r}\right), R\left(O_{t}, A_{r}\right)$, and $R$ be related through

$$
R^{4}=R^{2}\left(A_{t}, O_{r}\right) R^{2}\left(O_{t}, A_{r}\right) .
$$

Using the terminology introduced above, the general communication relation (3), may be cast in a form analogous to the radar equation

$$
\begin{aligned}
P_{r}= & \frac{P_{t}}{4 \pi R^{2}\left(A_{t}, O_{r}\right)} G\left(A_{t}, O_{r}\right) \\
& \cdot \frac{\sigma\left(A_{t}, O_{r}, O_{t}, A_{r}\right)}{4 \pi R^{2}\left(O_{t}, A_{r}\right)} A\left(O_{t}, A_{r}\right) .
\end{aligned}
$$




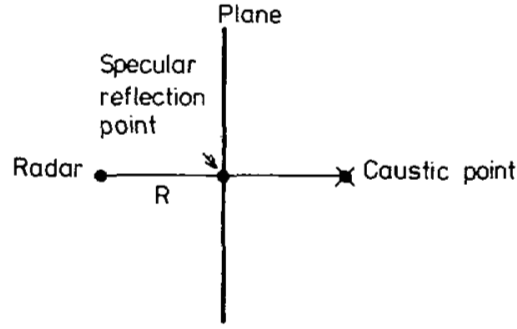

Fig. 3. Infinite plane problem.

This form shall now be adopted in order to illustrate two major principles in accordance with which centers $A_{t}, O_{r}, O_{t}$, and $A_{r}$ can be constructed. One principle is the radar principle in which $A_{t}=A_{r}$ and $O_{t}=O_{r}$. The other principle is the optics principle in which $A_{t}=A_{r}$, but $O_{r}$ may be different from $O_{r}$ as in geometrical optics. Because (8) may be used in accordance with geometrical optics, it is called the optics equation. Here, the two-way transmission problem is apparent from the presence of two distances.

\section{B. Infinite Plane}

For an infinite perfectly conducting plane illuminated by a radar located at a distance $R$ as shown in Fig. 3, $\pi R^{2}$ is given as the radar cross section by [13]. However from (1) is obtained

$$
\sigma=\lim _{R \rightarrow \infty} 4 \pi R^{2}
$$

since $\left|\bar{E}_{s}\right|=\left|\bar{E}_{i}\right|$ for the present case. Thus in accordance with [19] a near-field radar cross section $4 \pi R^{2}$ may be suggested.

It shall now be shown that $\pi R^{2}$ is natural to use when the radar principle is applied and that $4 \pi R^{2}$ is natural to use when the optics principle is applied. First, by considering transmission from the image of the radar in the plate to the radar itself, it is found that

$$
P_{r}=\frac{P_{t}}{4 \pi(2 R)^{2}} G A \text {. }
$$

Now, if the radar principle is used and $A_{t}=A_{r}$ and $O_{r}=O_{t}$ are chosen at the radar and the specular reflection point, rêspectively, i.e.,

$$
R\left(A_{t}, O_{r}\right)=R\left(O_{t}, A_{r}\right)=R \text {. }
$$

Equations (8), (10), and (11) constrain the near-field radar cross section to be $\pi R^{2}$ in accordance with [13].

If the optics principle is used and $A_{t}$ and $A_{r}$ are chosen at the position of the radar, but $O_{r}$ at the specular reflection point and $O_{t}$ at the image, i.e.,

$$
R\left(O_{t}, A_{r}\right)=2 R
$$

$(8),(10)$, and (12) constrain the near-field radar cross section to be $4 \pi R^{2}$ in accordance with [19]. It is seen that the radar principle considers the same path length for the incident field and the reflected field, while the optics principle uses different path lengths as in geometrical optics.

\section{Sphere}

For a sphere large in terms of wavelength, a near-field radar cross section can be found from (1) to be

$$
\sigma=\pi a^{2}\left(1+\frac{a}{2 R}\right)^{-2}
$$

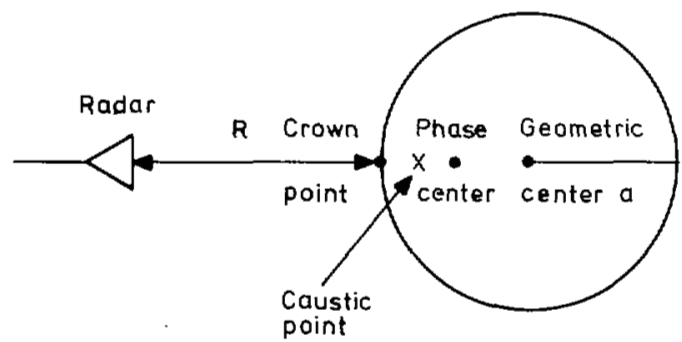

Fig. 4. Sphere problem.

where $a$ is the radius of the sphere, and $R$ is the distance from the crown point on the sphere to the field point [19], see Fig. 4. For $a \rightarrow \infty$ it is noted that $\sigma \rightarrow 4 \pi R^{2}$, i.e., the plate case. Therefore, it can be shown by making use of the optics principle that if $O_{r}$ is chosen at the crown point and if $O_{t}$ is chosen inside the sphere at the caustic point for reflected rays, a value for $P_{r}$ obtained from (8) will agree with a value of $P_{r}$ which can be found from simple ray tracing.

With reference to the result in (13), another near-field cross section

$$
\sigma=\pi a^{2}\left(1+\frac{a}{R}\right)^{-2}
$$

is given for large spheres [9]. For $a \rightarrow \infty, \sigma \rightarrow \pi R^{2}$. Again, since for $a \rightarrow \infty$ the sphere approaches a flat plate at the crown point, the radar principle can be used to show, if $O_{r}$ and $O_{t}$ are chosen at the crown point, the correct value of $P_{r}$ is obtained.

Applying the radar principle, it may also be shown that

$$
\sigma_{r}=\pi a^{2}
$$

can be used in the radar equation together with a distance

$$
R_{a}=\sqrt{R(R+a)} .
$$

From this result a more accurate procedure for the calibration of a scattering measurement set-up is derived. Since $\pi a^{2}$ is the geometrical optics contribution in the far field, it is concluded that the use of the corresponding apparent reflection center in connection with calibration will give more accurate results than the use of the geometric center, the specular reflection point, or the phase center of the sphere together with the far-field cross section. However it should be noted that since for $a \ll R$

$$
\sqrt{R(R+a)} \cong R+\frac{a}{2}
$$

the phase center is a good approximation for the apparent radar reflection center for many practical set ups for which $a \ll R$

It is also concluded that since the far-field radar cross section oscillates around the geometrical optics contribution, a more accurate determination of the apparent reflection center corresponding tc the far-field radar cross section oscillates around the points characterized by the parabola given by (17). In the case of a radar having a Hertzian dipole as antenna, the oscillations of the apparent reflection center is found by [14]. In this work use was made of a field solution of the problem presented by [18]. The oscillations around the parabola were demonstrated. 


\section{CONCLUSION}

It is demonstrated that centers of structures in electromagnetism depends on arbitrary choices. Several principles for finding phase, amplitude, and reflection centers are outlined. It is emphasized that uses of correction factors and near-field parameters in the Friis's transmission formula and the radar equation require specification of associated centers for the structures involved.

In particular, using the radar principle, an accurate reference point for the apparent reflection center of the sphere has been found. This can be used in calibration of a scattering measurement set up. As another example, by applying the minimum-number principle it should be possible to find a center of rotation around which it is most convenient to rotate antennas in near-field scanning techniques.

\section{ACKNOWLEDGMENT}

The author wishes to thank Torkild Linde-Hansen for reading and commenting on the manuscript.

\section{REFERENCES}

[1] J. Appel-Hansen, "Accurate determination of gain and radiation patterns by radar cross-section measurements," IEEE Trans. Antennas Propagat., vol. AP-27, no. 5, pp. 640-646, Sept. 1979.

[2] J. Appel-Hansen and R. J. King, "Experimental study of surfacewave propagation on a low-permittivity medium," Electron. Lett., vol. 15, no. 4, pp. 101-102, Feb. 15, 1979.

[3] J. Appel-Hansen, "Near-field cross sections and reflection centres for large spheres and plates-a fundamental analysis," Electromagnetics Institute, Technical Univ. Denmark, R 231, Aug. 1980.

[4] - "Antenna measurements," Electromagnetics Institute, Technical University of Denmark, vol. I and II, FN 36, Oct. 1980.

[5] L. J. Chu, "Physical limitations of omni-directional antennas," $J$. Appl. Physics, vol. 19, pp. 1163-1175, Dec. 1948.

[6] T. S. Chu and R. A. Semplak, "Gain of electromagnetic horns," Bell System Tech. J., vol. 44, pp. 527-541, 1965.

[7] R. E. Collin and S. Rothschild, "Evaluation of antenna $Q$," IEEE Trans. Antennas Propagat., vol. AP-12, no. 1, pp. 23-27, Jan. 1964.

[8] H. L. Crispin and J. S. Hollis, in Microwave Antenna Measurements, J. S. Hollis, T. J. Lyon, and L. Clayton, Ed. Atlanta, GA: Scientific-Atlanta, July 1970, pp. 9-14.

[9] J. W. Crispin and K. M. Siegel, Methods of Radar Cross-Section Analysis. New York and London: Academic, 1968, p. 388.
[10] J. D. Dyson, "Determination of the phase center and phase patterns of antennas," in Radio Antennas for Aircraft and Aerospace Vehicles, W. T. Blackband, Ed. Agard Conf. Proc., Slough, England: Technivision Services, 1967, no. 15.

[11] J. D. Hunter and I. G. Morgan, "Near-field gain correction for transmission between horn antennas," IEEE Trans. Instrum. Meas., vol. IM-26, no. 1, pp. 58-61, Mar. 1977.

[12] E. V. Jull, "Finite-range gain of sectoral and pyramidal horns," Electron. Lett., vol. 6, no. 21, pp. 680-681, Oct. 15, 1970.

[13] D. E. Kerr, Propagation of Short Radio Waves. New York: Dover, 1951 , p. 467.

[14] T. Linde-Hansen, "Near-field parameters for a perfectly conducting sphere," M.Sc. thesis, Electromagnetics Institute, Technical Univ. Denmark, Denmark, Aug. 1980.

[15] A. C. Ludwig, "Near-field far-field transformations using spherical-wave expansions," IEEE Trans. Antennas Propagat., vol. AP-19, no. 2, pp. 214-220, Mar. 1971.

[16] A. C. Ludwig, J. Hardy, and R. Norman, "Gain Calibration of a Horn Antenna Using Pattern Integration," Jet Propulsion Lab., California Institute of Technology, Pasadena, Tech. Rep. 32-1572, Oct. 1, 1972.

[17] A. C. Newell, R. C. Baird, and P. F. Wacker, "Accurate measurement of antenna gain and polarization at reduced distances by an extrapolation technique,"' IEEE Trans. Antennas Propagat., vol. AP-21, no. 4, pp. 418-431, July 1973 .

[18] P. Thomsen, "Back-scattering cross section of spheres used for calibration of radar test ranges," M.Sc. thesis, Electromagnetics Institute, Technical Univ. Denmark, Denmark, Jan. 1979.

[19] V. H. Weston, "Near-zone back-scattering from large spheres," Appl. Sci. Res., The Hague, Martinus Nijhoff, section B, vol. 9, pp. 107-116, 1962.

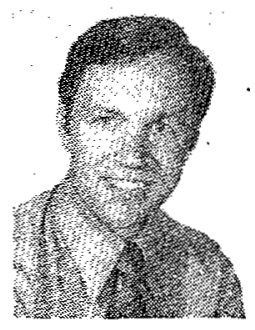

Jørgen Appel-Hansen was born in Farendløse, Denmark, on March 13, 1937. He received the M.Sc. degree and the Ph.D. degree in electrical engineering from the Technical University of Denmark, Lyngby, in 1962 and 1966, respectively.

In 1967 he was a Visiting Research Associate at the University of Michigan, Ann Arbor. From 1968 to 1978 he worked as head of the Radio Anechoic Chamber, Electromagnetics Institute. $\mathrm{He}$ is currently an Associate Professor at the Electromagnetics Institute. He has organized several international postgraduate courses in antenna and scattering measurements. He is also a consultant in design of anechoic chambers, test ranges, and their instrumentation and implementation of measurement procedures. 